\title{
An Intelligent Monitoring System for Electric Based on WSN
}

\author{
Jianjun $\mathrm{YI}^{1, *}$, Zhaoqing XIONG, Baiyang $\mathrm{JI}^{2}$, Xiaomin $\mathrm{ZHU}^{1}$, Jun XU ${ }^{1}$ \\ ${ }^{1}$ Department of Mechanical Engineering, East China University of Science and Technology, Shanghai \\ 200237, China \\ ${ }^{2}$ Hangzhou Sunyard System Engineering Co., Ltd., Hangzhou 310053, China \\ "Corresponding author
}

\begin{abstract}
With the rapid development of information technology and the internet of things, the future electric equipment not only needs to monitor power state but also needs to be able to be dispatched and remotely monitored. In order to adapt to this trend, we develop a smart monitoring device based on wireless sensor network (WSN).which consists of smart sockets, handheld monitoring terminal and remote control part (PC).The sockets send real-time datum to the terminal by wireless. Then control terminal receives, stores and displays these information and can transmit them to the PC by the TCP/IP protocol. The PC also can indirectly control the sockets through the terminal. The load balancing strategy that embedded in the device can realize the reasonable use of the electricity. Considering the foreign demand, this device can be used in different countries. Finally, this equipment also adapts to the demand of intelligent home.
\end{abstract}

KEYWORD: smart meter; WSN; monitoring; load balancing

\section{INTRODUCTION}

Power is inseparable in people's lives, but with the development of technology the current meter has been increasingly unable to meet people's demand[1]. Firstly, the traditional mode is inconvenient to realize human computer interaction. We can only read the total power consumption by old mode which can't reflect the power consumption in details and can't record and store consumption by monthly, daily and hourly. It also can not check how much consumption every equipment consumes. So the users can not understand why the appliances use so much energy[2]. Meanwhile the traditional mode do not own foundation of remote control and supervision, so it is behind the times of intelligence.

Secondly, the power management is too inconvenience. Residential electricity exist valley and peak at different times in different seasons. Usually, we solve these problems by limiting and cutting off the power[3]. We also call on residents to improve awareness of saving electricity, but the awareness is relatively weak and the effect is less pronounced. Finally, the intelligent is too low. People still occupy the dominant position in the traditional mode which will bring a series of problems. Operations like manual meter require a lot of manpower and resources. At the same time the risk of error is also significantly improved. These problems widely exist in the domestic and foreign.
This predicament is in sharp contrast to high degree of automation in modern which is a major obstacle to development of the power supply system[4]. Therefore it is necessary to improve traditional electricity meter to conform to the needs of modern social development.

Wireless sensor networks(WSN) whose one of key technologies is wireless communication is with the features of large-scale, self-organization, dynamic, reliable, data-centric and so on. In communication, wireless sensor networks don't require very high communication bandwidth and communication rate. It costs lower power consumption and delay is shorter. At same time WSN can also ensure good network selforganization and communication security. In MSN the ZigBee technology with high efficiency, high reliability, high security, high self-organizing network, low-power, low-rate, low complexity, low cost and so on makes it to be the preferred communication protocol and used in many fields[5]. At the same time wireless sensor module can be compatible with other modules. So it will be a reality that WSN is used in the electricity and the intelligence network will be further realized.

Therefore, this paper of the intelligent monitoring systems in power based on WSN has the following advantages.

(1) It has a lower requirement for space and can increase scalability of the internal network. 
(2) ZigBee used as the internal network mode with the features, such as low cost, low power consumption, high reliability and so on can achieve normal communication up to $200 \mathrm{M}$ without blocking and can realize automatic network up to or more nodes.

(3) The terminal with simple structure, easy usage and embedded system of $\mu \mathrm{C} / \mathrm{OS}$ and GUI meets the demands of real-time requirements, interactive interface and manual control.

(4) The device with TCP/TP communication can realize remote control and collect data which lays a solid foundation for deeper data analysis.

(5) The device embedded Smart power scheduling strategy which can save energy and reduce the cost. Especially this way can effectively avoid the peak and valley of using power.

The main content of this paper is how to develop the device of intelligent monitoring based on WSN and test this device. We will describe our work as the following. Firstly, we will briefly describe the structure of this device. And then, we will analyze how to design the hardware and software. At last, we will test this device.

\section{SYSTEM ARCHITECTURE}

The overall system structure, shown in Figure 3-1, includes two intelligent sockets with child node of ZigBee, one control terminal with coordinator of ZigBee and remote communication section.

\subsection{Internal network of electrical equipment}

The internal network includes sockets and terminal that they communicate with each other via ZigBee.

In the internal network, electrical devices plugged in the smart socket that they can calculate the current power value and send these information to the control terminal by ZigBee. In the socket, we also integrate switch control module, so we can realize turning on and off the sockets.

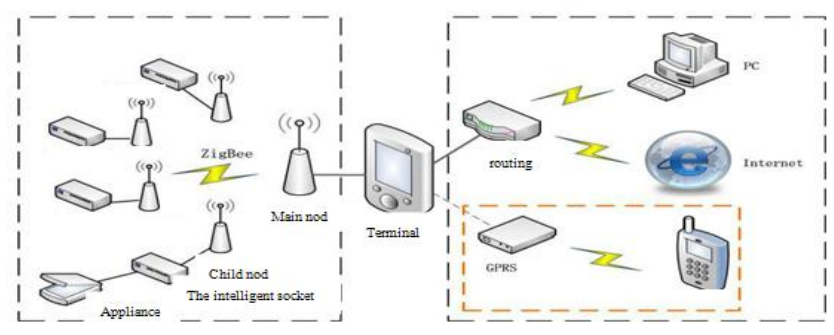

Fig. 2-1 Overall architecture Figure

\section{(1) Hand-held control terminal}

Hand-held control terminal is a key part in the whole devices. It embeds the ZigBee modules and works as a controller to the internal network, a server to the PC.

\section{(2) Remote communication terminal}

The remote $\mathrm{PC}$ and control terminal communicate with each other via TCP/IP protocol. By Web technology, the information can orderly transmit between the browser and server based on standard protocol. It is different from the traditional client/server models that it doesn't need to install specific client application on the client computer. we can realize functions as requirements by web browser. Such as inquiring and getting information, turning on and off the sockets and so on.

\section{HARDWARE}

Hardware includes two parts - intelligent sockets and control terminal.

\subsection{Intelligent sockets}

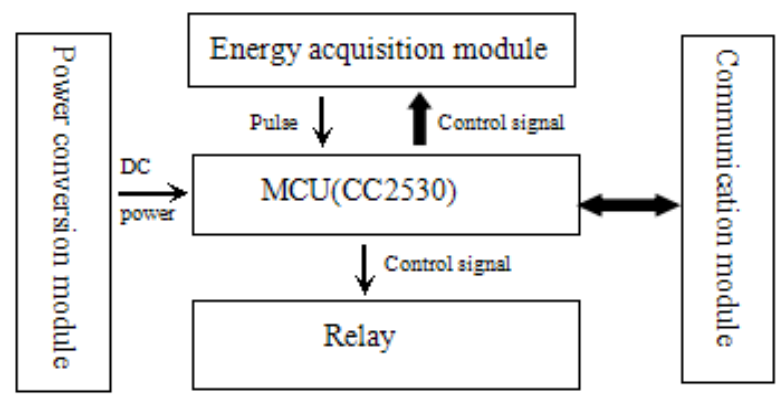

Fig. 3-1 Socket's hardware

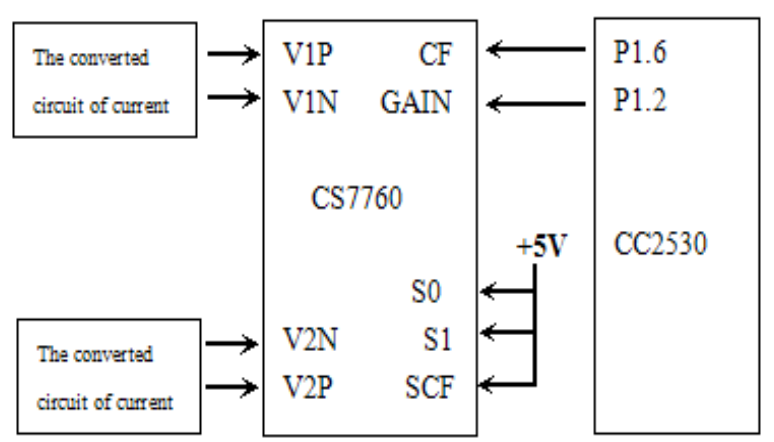

Fig. 3-2 CS7760 and CC2530

The sockets is a part of data acquisition in the whole device that it can also realize the switching operation of the electrical equipment and it also is the object operated by terminal. After powered on, the sockets are always in working state and acquire the value of power. When the coordinator sends the orders, the sockets will conduct corresponding functions as ordered, such as turning on or off the Appliances, acquiring the value of power. And so on.

As functions, the hardware design of intelligent sockets, shown in Figure 3-1, mainly includes the following modules, such as power, the relay, 
electrical energy collection, ZigBee and so on. The core chip is CC2530 of TI[6].

We can achieve the following functions by the Power conversion module. The DC $12 \mathrm{~V}$, converted from AC $220 \mathrm{~V}$ by the LNK304, will be converted to DC $5 \mathrm{~V}$ by 78L05 and finally will be converted to DC $3.3 \mathrm{~V}$ by 7533 . These DC power will be supplied to the Appliances.

The relay module is used to achieve switch control. We turn on or off the relay by controlling the voltage of base potential of the 8050 transistor. When the voltage of base is high, the relay is turned on. When the voltage is low, the relay is turned off.

Energy acquisition module whose core chip is CS7760 which is a high precision energy acquisition chip. This chip will convert the power to the digital pulses. The CC2530 calculates the value of power based on the pulses. The module is shown in Figure 3-2.

ZigBee module is designed based on CC2530. When the ZigBee modules receive the signal from the terminal, it will analyze these information and then conduct the related foundation, such as calculating power, turning on or off the switch and so on.

The socket is designed with the European Standard for flexible interface. It will be compatible with the foreign equipment under the help of power converter.

\subsection{The terminal}

The terminal is very important in the whole devices. On the one hand it queries and controls the intelligent sockets, the other hand it is queried and controlled by the remote PC. The terminal, shown in Figure 3-3, includes the following parts, such as MCU part, power conversion input module, EEPROM, touching screen module and so on. It also includes three communication modules that is ZigBee, RS232 and TCP/IP communication.

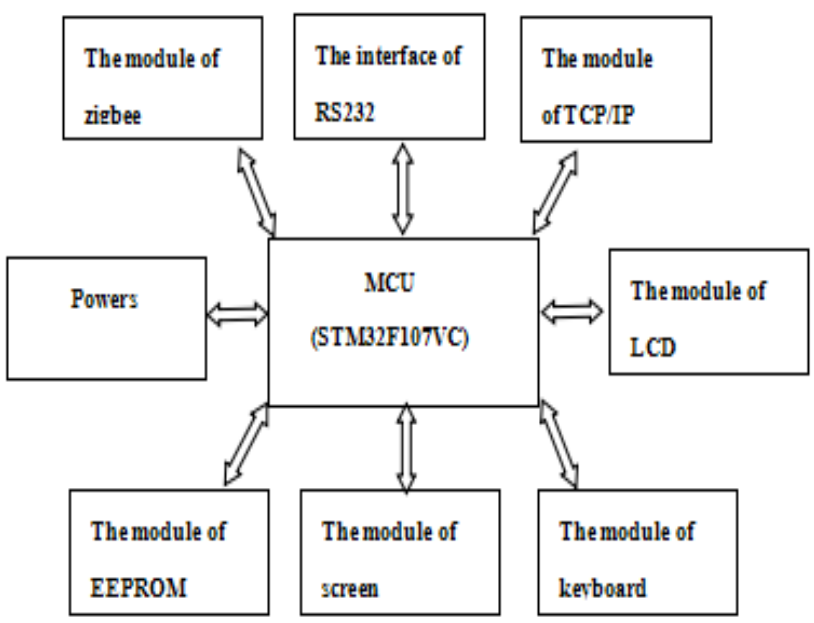

Fig. 3-3 Control terminal's hardware
The terminal's MCU is STM32F107VC that is connected with the ZigBee module by UART. The ZigBee is configured as the coordination which is the main controller in the whole network of ZigBee.

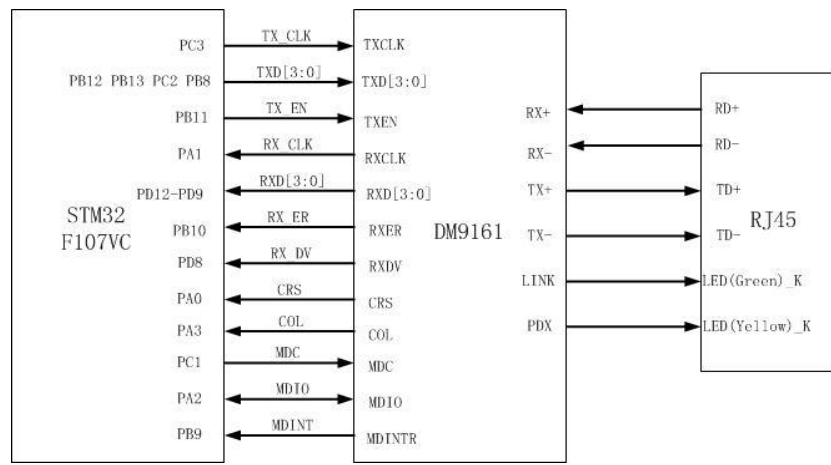

Fig. 3-4 Network module's interface

Although there are rarely $300 \mathrm{k}$ storage in the MCU, We still add an EEPROM module to store critical datum to avoid losing the datum after power outage.

The terminal supports the popular LCD touching screen in the market. The device will be colorful through the screen. We not only design the matrix keyboard control module in hardware which can connect with a $4 \times 5$ matrix keyboard conveniently, but also design the keyboard in the screen. It is very useful for this devices.

In communication, we design a network communication module to realize remotely accessing and controlling the terminal by TCP/IP. The module includes DM9161 (an Ethernet PHY chip),RJ45 network port HR911105A devices and so on. MCU connects with DM9161 via MII interface and external network communication via HR911105 interface, shown in the Figure 3-4. In addition, we also design a RS232 module system debugging and it could also be used as a reserved interface for system expansion.

\section{SOFTWARE}

Software mainly includes the program in three parts that is sockets, terminal and remote PC. The overall process is shown in Figure 4-1.

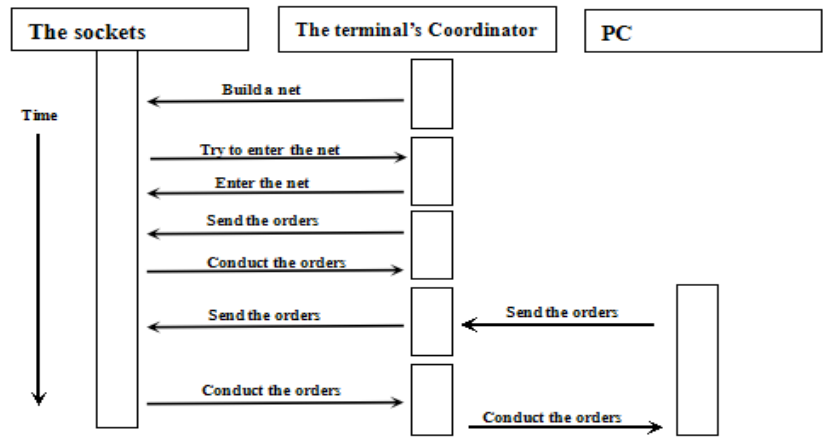

Fig. 4-1 Overall control process 
The sockets mainly collect the datum and its software is relatively simple. After powering, the system start running and completes the following functions in turn, initializing socket, ZigBee joining in the network, collecting power, receiving and analyzing orders and executing the order. The order has two kinds. The one is turning on or off sockets. The other is to order the socket to send data to the terminal. The detail process is shown in Figure 42.The program is used to convert pulse to the power value, shown in the Figure 4-3.

The running process of terminal is shown in Figure 4-4.After powering, the entire system starts running. Firstly, the task scheduling based on $\mu \mathrm{C} /$ OS is initialized. Every task is run as their respective priority. The monitoring screen is run and then devices wait the operation of user or task is occurred. When the key is pushed, the system conducts corresponding operation. In the system, there are multiple tasks, such as displaying interface, sending and receiving datum, accessing the internet, restoring datum, analyzing datum, balancing the power and so on. The terminal sends the order of inquiring power in every minute. At same time we also embed the strategy of balancing the power in order to avoid the peak of using the energy.

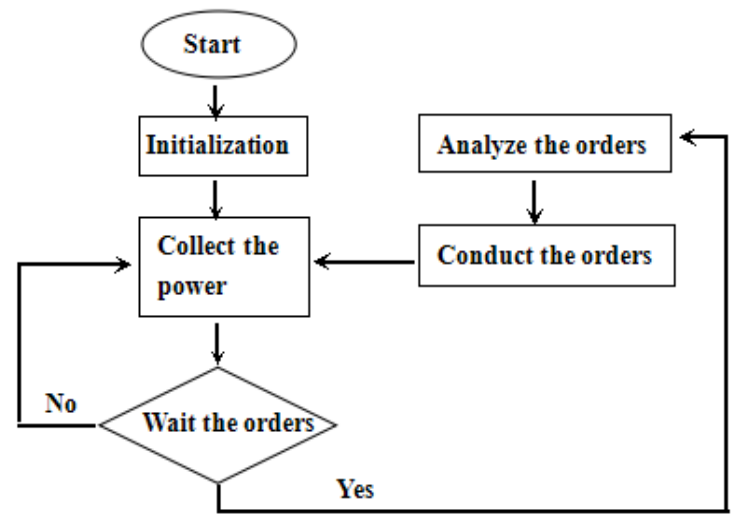

Fig. 4-2 Smart socket software flow chart

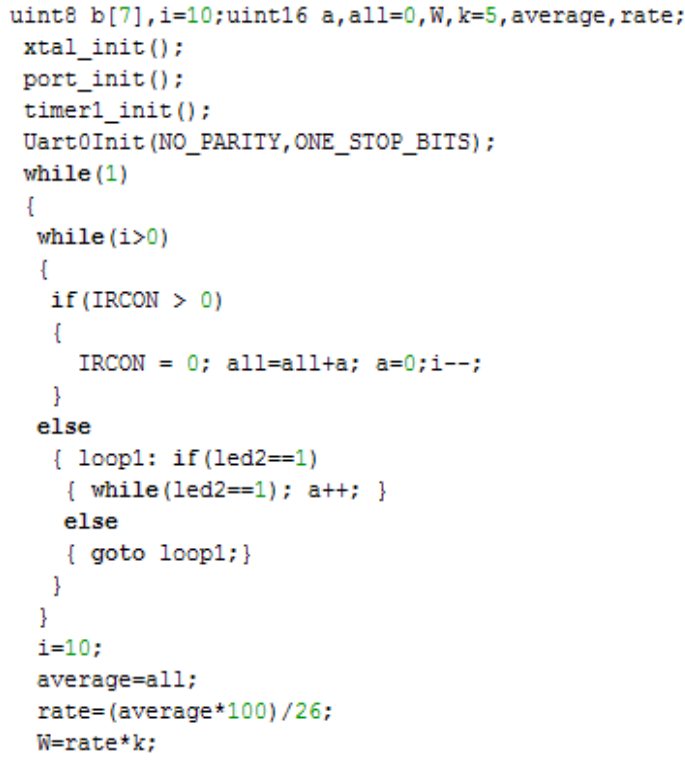

Fig. 4-3 Electrical energy into the program

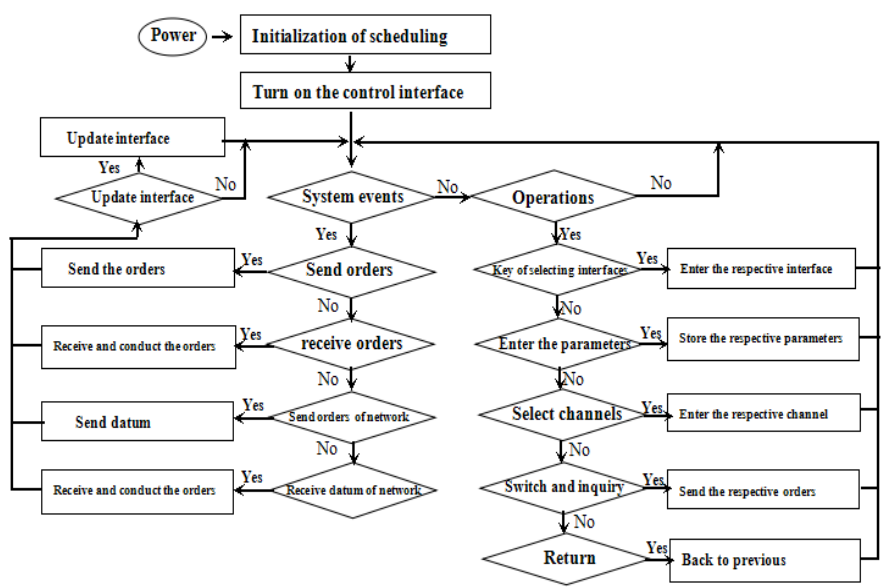

Fig. 4-4 Terminal software flow control

The program of PC is used to realize remotely inquiring and controlling the appliances of residents as the protocol of TCP/IP. People can scan the realdatum from the terminal and send orders to the terminal to indirectly control the sockets by Web browser. We realize this function by $\mathrm{C} \#$ program based on TCP / IP Socket.

\section{TESTING AND VALIDATION}

\subsection{Overall testing and validation}

This paper's object is to develop an intelligent monitoring device of power based on WSN. The above describes the hardware and software of device in detail. Now we will test this device.

This device includes a terminal, three intelligent sockets, Jlink simulation, a table lamp as an electrical equipment and other parts which are not shown in the paper. The lamb is $25 \mathrm{~W}$.Now we will briefly show this device.

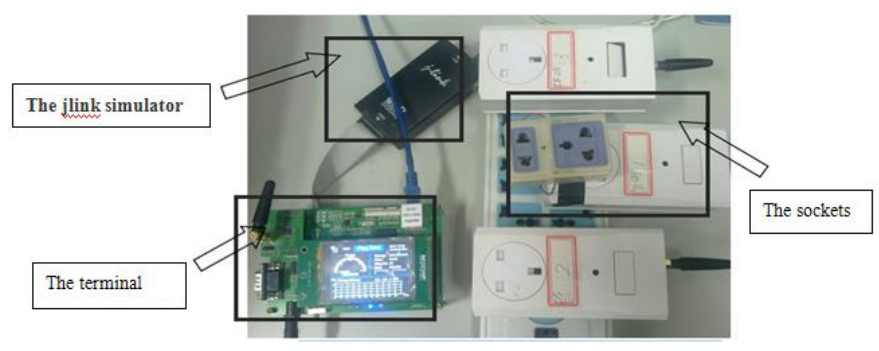

Fig. 5-1 The testing device

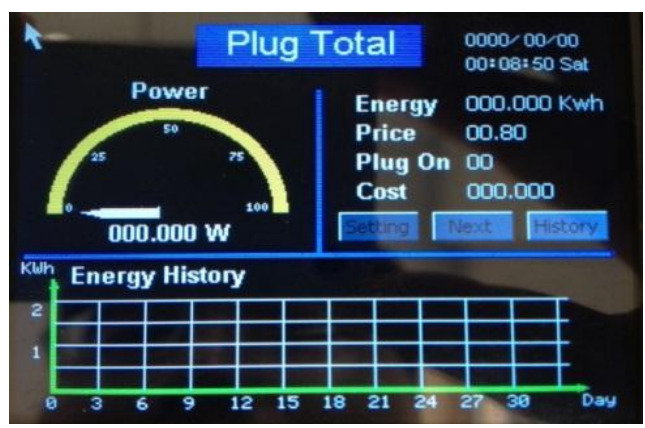

Fig.5-2 Terminal initial interface 


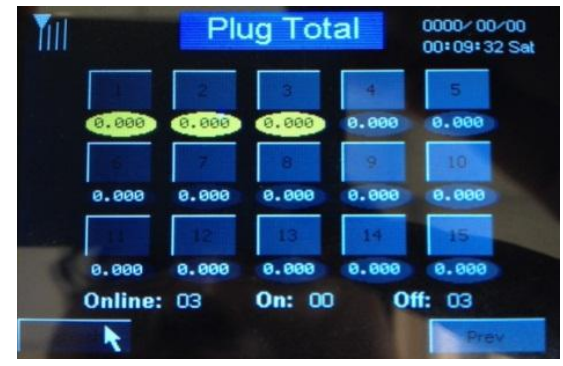

Fig.5-3 Total nod button interface

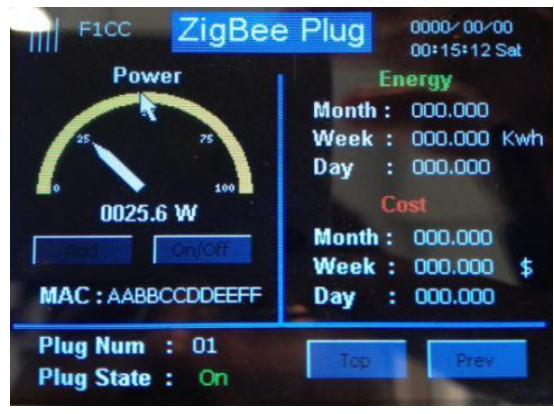

Fig. 5-4 Child-nod interface

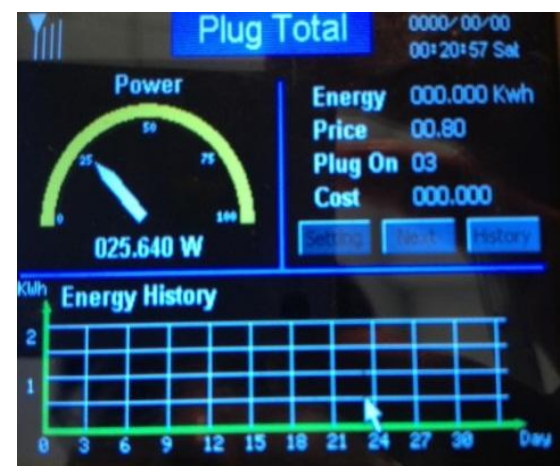

Fig. 5-5 Main interface

When we power the terminal, it will show the initial screen, shown in Figure 5-2.In the screen we scan the total value of power. Because the sockets is off, the value is $0 \mathrm{~W}$.Then we will push the 'next' button to enter the total button interface, shown in Figure 5-3.The yellow represents corresponding socket. Now there are three sockets, so number of yellow is three. Pushing down the three button and turning on the switches. Then we scan the first child interface and show the

Corresponding value .The value of first child is shown in Figure 5-4.The value of first child which we get from the Web in the PC is shown in Figure 56 .We can find that this device is very reliable after comparing these value.In the end we turn off all the sockets. This is the brief device testing.

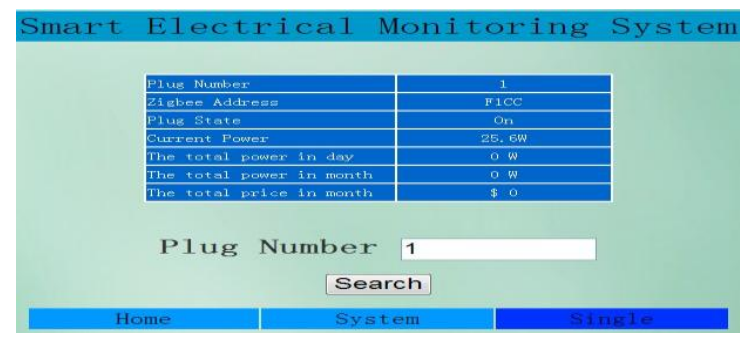

Fig. 5-6 The first node state of the PC
In addition to these, there are more functions. For example in the terminal there are functions of power consumption records of each month, the total cost, chart with 'electricity - date', parameter configuration and removing or adding the nodes and so on. The PC can also turn on or off the sockets, record the consumption, account the cost, inquiry the objective nods, draw the chart and so on. Because the content is too more, we just show its basic function.

\subsection{Accuracy testing}

Accuracy of the value of power is a very important parameter. It directly determine the reliability and usefulness of the device. So it must be tested firstly.

Due to the limited experimental conditions, we design a following test mode. Three kinds of lambs are correspondingly plugged in the three sockets that the power of lambs is respectively $10 \mathrm{~W}, 40 \mathrm{~W}$ and $50 \mathrm{~W}$.We read the values of power from the terminal and compare these datum. We can get the accuracy from the following 10 sets of data, shown in table5.1. The testing conditions is following.

(1) Previously correct the value of power.

(2) The device run 5 minutes before testing.

(3)The range of power isn't beyond 100W.

(4) Previously test the value of power without load.

The datum show the following information. The power of consumption is rarely $0 \mathrm{~W}$ without load and the most value is $0.3 \mathrm{~W}$. The range of error is $2.5 \%$ to $3 \%$.Although the device has some error that the sockets itself need consuming some power, it can accurately reflect the situation of appliances. This device has some practical significance.

Table 5.1 Accuracy relative value

\begin{tabular}{|c|c|c|c|c|}
\hline No. & Power(0W) & Power( 10W) & Power(40W) & Power(50W) \\
\hline 1 & 0.2 & 10.1 & 40.5 & 51.1 \\
\hline 2 & 0.1 & 10.2 & 39.2 & 50.8 \\
\hline 3 & 0.0 & 9.8 & 39.6 & 49.4 \\
\hline 4 & 0.2 & 9.9 & 40.3 & 50.1 \\
\hline 5 & 0.3 & 10.1 & 40.8 & 50.8 \\
\hline 6 & 0.2 & 10.1 & 39.8 & 50.6 \\
\hline 7 & 0.2 & 9.9 & 40.2 & 51.3 \\
\hline 8 & 0.3 & 10.0 & 40.3 & 51.2 \\
\hline
\end{tabular}

*T.V. means: theoritical value; A.V. means average value; Max. R.E. means maximum

\subsection{Distance testing - No obstacle}

The most distance of testing is $200 \mathrm{M}$ and there is a testing position in every $10 \mathrm{M}$.We check the data from the sockets to judge the communication distance when the terminal send the order of inquiring power. 
The testing conditions:

(1) The terminal and sockets are generally placed.

(2) There are no obstacle between terminal and sockets.

(3) We test 10 times in every position and each operation interval is more than 5 seconds.

(4) Exclude the abnormal results.

We can get information from the above table. On the one hand, the most distance between terminal and sockets is $120 \mathrm{M}$. When the distance is over the $120 \mathrm{M}$, the quality of communication is rapid decline. The other hand, this device's best communication distance is within $100 \mathrm{M}$.

Table 5.2 Communication distance (No obstacles)

\begin{tabular}{|c|c|c|}
\hline Com. Dist.(m) & Rec. num. & not-Rec. num. \\
\hline 0 & 10 & 0 \\
\hline 10 & 10 & 0 \\
\hline 20 & 10 & 0 \\
\hline 30 & 10 & 0 \\
\hline 40 & 10 & 0 \\
\hline 50 & 10 & 0 \\
\hline 60 & 10 & 0 \\
\hline 70 & 10 & 0 \\
\hline 80 & 10 & 0 \\
\hline 90 & 9 & 1 \\
\hline 100 & 10 & 0 \\
\hline 110 & 9 & 1 \\
\hline 120 & 9 & 1 \\
\hline 130 & 8 & 2 \\
\hline 140 & 8 & 2 \\
\hline 150 & 8 & 2 \\
\hline 160 & 5 & 5 \\
\hline 170 & 2 & 8 \\
\hline 180 & 0 & 10 \\
\hline 190 & 0 & 10 \\
\hline 200 & 0 & 10 \\
\hline
\end{tabular}

\subsection{Distance testing - Through the wall}

In the lab environment, we test the information quality from passing the one to three walls. When the terminal sends the orders of inquiring the power, we check whether the sockets can send the answers to the terminal. Then we judge the quality of communication under the obstacle by the situation of answers.

The testing conditions:

(1) The wall is ordinary brick wall and its thickness is $24.5 \mathrm{CM}$.

(2) The terminal and sockets is separated by this wall.
(3) The distance between terminal and wall, sockets and wall is $1 \mathrm{M}$.

(4) The distance between wall and wall is $4 \mathrm{M}$.

(5) We test 10 times in every position and each operation interval is more than 5 seconds.

(6) Terminal and sockets is generally placed.

Table 5.3 Communication distance - through the wall

\begin{tabular}{|c|c|c|}
\hline Nums. of walls & Not-Rec. num. (\%) & Rec. num. (\%) \\
\hline 1 & 10.3594 & 64.4531 \\
\hline 2 & 10.1719 & 65.6406 \\
\hline 3 & 10.3438 & 63.3750 \\
\hline
\end{tabular}

From the table, we can see the following information. The signal can successfully pass onelayer wall. When passing two-layer walls, the signal's quality rapidly declines. When passing three-layer wall, the device can't communicate with each other. Therefore this device can normally communicate with each other when passing through one-layer wall. But when the layer of wall is over one, the communication will is effected. It is worth mentioning that we will develop this device to various communication modes, such as the tree or mesh topology. The biggest advantage of these modes is that it can achieve network expansion through routing. So this development can avoid or reduce the situation that the signal must pass through the wall.

\section{SUMMARY}

This paper mainly describe how to develop the intelligent monitoring equipment based on WSN. This device can realize monitoring and managing the residents' appliances. In the paper we describe the overall structure, hardware, software and testing. So we basically design a power monitoring equipment based on ZigBee.

This device not only increase the automation of power equipment and reduce costs, but also improve the efficient operation and home security. At the same time, along with the power shortage, the development of wireless communication technology, lower chip prices, regulatory requirements, the radio can monitor system own a good economic and promotion prospect. This device not only can be applied to the family, but also can be further extended to the building monitoring, wireless meter reading, industrial plant monitoring and a series of fields.

Although this device has been basically designed, there are still some deficiencies, for example the study of load balancing is not perfect. The communication distance is short. The consumption and cost are not accounted. The packaging isn't designed, and so on. We need to solve these 
problems step by step in later studies. It is believed that this design will be more widely applied in the future.

\section{ACKNOWLEDGMENTS}

This paper is supported by the Research Foundation of Science and Technology Commission of Shanghai under Grant No. 10DZ1500200, the Nature Science Fund of China (NSFC) under Grant Nos: 50975088, 51275173, 51210105018, the Fundamental Research Funds for the Central Universities under Grant No. WH0913009, Shanghai Pujiang Program under Grant No.PJ201000353, Shanghai Software and IC industry Development Special Fund under Grant No. 120493.

\section{REFERENCES}

[1] Zhiyang Lu. The development of power monitoring system based on wireless technology. Electronic Testing Technology, 2011, 34(08): 99 100.

[2] Xinhua Yang and Peng Wang. The design of remote automatic meter reading system base on ZigBee, Electric Automation, 31(05): 45-47.

[3] X.Xi. C.Tao. and X.Fang.A health care system based on PLC and ZigBee. Wireless Communication, Networking and Mobile Computing, Shanghai, CN.Sep.21-25, 2007, pp.3063-3066.

[4] Shuchao Ma, Ming Zhu and Lei Wang. A Remote Monitoring System of IDC Room Based on ZigBee Wireless Sensor Networks. 2009, 8th IEEE Internation Symposium on Dependable, Autonomic and Secure Computing, DASC 2009, P 782-787.

[5] Zhongzhong Li and Yuchao Duan.Advanced applications of CC1010 wireless SOC. Beijing: The press of BeiHang University, 2007: 30-45.

[6] The datasheet of CC2530. 